\title{
Visual mismatch negativity and stimulus-specific adaptation: the role of stimulus complexity
}

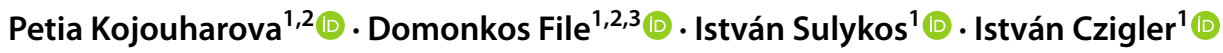

Received: 18 June 2018 / Accepted: 15 February 2019 / Published online: 26 February 2019

(c) The Author(s) 2019

\begin{abstract}
The present study investigated the function of the brain activity underlying the visual mismatch negativity (vMMN) eventrelated potential (ERP) component. Snowflake patterns (complex stimuli) were presented as deviants and oblique bar patterns (simple stimuli) as standards, and vice versa in a passive oddball paradigm. Control (equiprobable) sequences of either complex shape patterns or oblique bar patterns with various orientations were also presented. VMMN appeared as the difference between the ERP to the oddball deviant and the ERP to the control (deviant minus control ERP difference). Apart from the shorter latency of the vMMN to the oblique bar pattern as deviant, vMMN to both deviants was similar, i.e., there was no amplitude difference. We attributed the function of the brain processes underlying vMMN to the detection of the infrequent stimulus type (also represented in memory) instead of a call for further processing (a possibility for acquiring more precise representation) of the deviant. An unexpected larger adaptation (control minus standard ERP difference) to the snowflake pattern was also obtained. We suggest that this was due to the acquisition of a more elaborate memory representation of the more complex stimulus.
\end{abstract}

Keywords Visual mismatch negativity $\cdot$ Automatic deviant detection $\cdot$ Stimulus-specific adaptation $\cdot$ Stimulus complexity

\section{Introduction}

The detection and identification of the changes in the visual environment are essential tasks of the perceptual system. A part of the change detection mechanisms are the brain processes underlying an event-related potential (ERP) component within the 120-350 ms post-stimulus latency range termed the visual mismatch negativity (vMMN). VMMN is elicited by visual events that violate a regularity in a stimulus sequence, even if the eliciting stimuli are unrelated to

Electronic supplementary material The online version of this article (https://doi.org/10.1007/s00221-019-05494-2) contains supplementary material, which is available to authorized users.

Petia Kojouharova

kojouharova.petia@ttk.mta.hu

1 Institute of Cognitive Neuroscience and Psychology, Research Centre for Natural Sciences, Hungarian Academy of Sciences, P.O. Box 286, Budapest 1519, Hungary

2 Doctoral School of Psychology, Eötvös Loránd University, Budapest, Hungary

3 Institute of Psychology, Eötvös Loránd University, Budapest, Hungary the ongoing behavior. In experimental context, vMMN is usually investigated in the passive oddball paradigm. In most cases the participants perform a visual task, while visual events are presented simultaneously outside its context as unattended stimuli. The unattended events form a stimulus sequence that sets up some sort of regularity: The stimuli could be identical, come from the same category, or follow a more complex sequential regularity. The stimuli conforming to the regularity are the standard stimuli. The sequence is infrequently interspersed with deviant stimuli that may differ by a visual feature (color, orientation, direction of movement, shape, spatial frequency, etc.), belong to a different category (e.g., a different facial emotion), or violate the sequential regularity (e.g., an irregular repetition within a sequence of alternating stimuli) (for reviews see Kimura et al. 2011; Kremláček et al. 2016; Stefanics et al. 2014).

VMMN is a counterpart to the more frequently investigated auditory mismatch negativity (MMN) (for a review on auditory MMN see Näätänen et al. 2011), so it is not surprising that the same theoretical explanations are applied as to the function of the underlying brain processes. At present there are two accounts concerning the elicitation of both (auditory) MMN and vMMN: The trace mismatch theory 
(see Näätänen 1992; for a critical overview see; Winkler 2007) and the predictive coding theory (e.g., Friston 2010). The aim of the present study is to investigate the function(s) of the brain activity underlying vMMN by presenting standard and deviant stimuli that are highly different both in visual complexity and categorically in a passive oddball paradigm. In this case, the two accounts make different predictions about the emergence of vMMN.

According to the trace mismatch theory, the sensory memory representation (trace) of the standard is compared to the representation of the incoming event. In the case of a difference (mismatch), the emerging brain activity is a correlate of a call for further processing of the deviant event (the possibility that an event is processed at a deeper level, i.e., more precise processing of orientation, or in the case of meaningful stimuli, the meaning per se, and/or the specificity of the event within the category). This account is related to the orientation theory proposed by Sokolov (1963), together with the notion that due to the continuous task-related processing and/or due to the deviancy not being sufficiently salient, other aspects of the orienting reaction (changes in the autonomic nervous system activity, motor processes) are absent (for a detailed explanation see Näätänen 1992). However, it was demonstrated that the violation of complex regularities [e.g., short tones are followed by low tones, and long tones are followed by high tones (Paavilainen et al. 2007)] also elicits MMN without being limited to a specific parameter of the standard stimulus, therefore MMN was considered to be an index of detected violation of any registered regularities within the stimulus sequence (e.g., Winkler 2007).

The predictive coding theory, a more recent account regarding the underlying mechanism of both (auditory) MMN and vMMN, proposes that to minimize the use of energy resources, "biological systems should continually minimize their surprise about sensory states" (Auksztulewicz and Friston 2016, p 126). According to this account, mismatch components are error signals. The function of the processes underlying both (auditory) MMN and vMMN is the adjustment of the representation of incoming stimulation to the representation of the model of the recent environment, i.e., a cascade of processes until the error is eliminated (for reviews see Garrido et al. 2009; Stefanics et al. 2015). In the auditory modality a large body of research supports the predictive coding view (for a review see Bendixen 2014). In contrast, the evidence for a similar interpretation of vMMN is based on a small set of studies. These studies investigated the ERP effects of violated sequential regularities with no specific parameter attached to the standard and the deviant. Such studies include the deviant repetition of a stimulus in a sequence of alternating stimuli pairs (Czigler et al. 2006), the deviant repetition of emotion in a sequence of alternating emotions (Kimura et al. 2011), the second (deviant) member of pairs of dots with different colors among pairs of dots with the same color (Stefanics et al. 2011), the second standard after a deviant (Kimura et al. 2010), and the violation of a rotating rule (Kimura and Takeda 2015), all of which elicited a posterior negativity (vMMN).

Thus, there are two different assumptions about the function of the brain activity underlying (auditory) MMN and vMMN. According to the trace mismatch theory, vMMN is the result of an automatic and putatively partial identification of the deviancy, and the role of the processes underlying this ERP component is to promote a more elaborate processing of the eliciting event. The predictive coding account, on the other hand, hypothesizes another locus within the stream of information processing-vMMN is the correlate of elementary identification of the incoming events not just as a deviancy, but also what deviancy within the environmental model.

In the present study we presented oddball sequences with simple stimuli (oblique bar patterns) and complex patterns (snowflake patterns). In one condition the oblique bar pattern was the standard and the snowflake pattern was the deviant, and in the other their roles were reversed. The choice of highly different stimuli was deliberate to prevent model adjustment, and thus make it possible to compare the two accounts. In other words, the emergence of vMMN in these sequences would be difficult to attribute to it being the correlate of the adjustment of an environmental model to the incoming activity (a putative cascade of processes leading to the matching of bottom-up and top-down processes) as supposed by the predictive coding theory as it is difficult to imagine that the presence of a deviant snowflake modifies the representation of an oblique bar or vice versa. However, if vMMN is an index of the mismatch between the representation of standard and that of the deviant at an initial stage without the involvement the adjustment process and the mismatch at subsequent stages of the cascade of processes, the putative mechanism underlying vMMN is fairly similar to that posited by the trace mismatch account. In addition, within an orientation-related theory such as the latter account one may expect that a more salient deviant elicits a more robust vMMN (defined here as a larger amplitude and/or a shorter latency).

VMMN is often calculated as the difference between the ERP responses to the deviant and the standard stimuli (deviant minus standard). However, in the oddball paradigm, the stimulus-specific adaptation (SSA, see below) difference between the negative exogenous ERP components (mainly the $\mathrm{N} 1$ component) to the frequent (standard) and rare (deviant) stimuli may contribute to the deviant minus standard difference potentials. A goal of our experimental design is to isolate the genuine mismatch response. Therefore, we included equiprobable control sequences. In the equiprobable control sequences the probability of the (randomly presented) various stimuli is identical to the probability of the 
oddball deviant. Consequently, in the control sequence no specific rule can be violated by any of the stimuli (Jacobsen and Schröger 2001; Schröger and Wolff 1996). Accordingly, the deviant minus control difference is free of SSA, i.e., it is a genuine mismatch response. When applied in the strictest sense, the additional stimuli in the equiprobable control sequence should be outside the range of the standard and deviant, beyond the critical deviant parameter. In certain cases (e.g., the pitch of auditory stimuli) the standard-deviant range can be defined reasonably. This is because the tonotopic representation in the auditory cortex corresponds to the spectrum of tone frequencies. However, there is no continuum between the oblique bar and the snowflake patterns, and we cannot apply the equiprobable control in a strict way. Here, we presented an equiprobable sequence with oblique bar patterns of various orientations, and another sequence with various complex shapes (for a review of vMMN studies using the equiprobable control procedure see File et al. 2017).

The investigation of SSA (decreased activity to stimulus repetition) is not the focus of the study, but it should be addressed. SSA is well-known at various levels of brain activity, behavioral effects, and subjective experiences, from single cell recording (Sawamura et al. 2006) to conscious experience (Clifford et al. 2010; Gibson 1937; Krekelberg et al. 2006) and has also been labeled as refractoriness or habituation (for a discussion see O'Shea (2015)). It is considered to be a consequence of memory acquisition or the result of memory update (e.g., Gonsalves et al. 2005; GrillSpector et al. 2006; Lafontaine et al. 2016; Sayres and GrillSpector 2006). Including equiprobable sequences in the design of the present study allows not only for isolating the genuine $\mathrm{vMMN}$, but also for addressing the presence of SSA computed as the control minus standard difference (Kimura 2012), even if the paradigm is not optimal for investigating SSA. This part of the study was exploratory.

In addition to the posterior region where $\mathrm{vMMN}$ is expected to emerge, frontal and central areas were also included in the analysis with exploratory purposes as there is an indication that there is a vMMN generator in the frontal areas (e.g., Kimura et al. 2010).

To sum up, there are three predictions regarding vMMN. First, a mismatch mechanism whose function is the mere indication of the deviancy as suggested by the trace mismatch theory predicts a similar vMMN in the conditions with complex and simple deviant stimuli. Second, a more salient deviant is expected to elicit a larger vMMN if this component is related to a call for further processing, i.e., an orienting reaction is initiated. Last, the predictive coding view does not rule out the emergence of vMMN, but the cascade of model adjustment is highly improbable in the present design, so in this case vMMN is not expected to emerge as a long-lasting negativity, i.e., in the 200-300 ms range.

\section{Methods}

\section{Participants}

Nineteen students [12 females; mean age 22.7 years $(\mathrm{SD}=2.39$ years $)]$ participated in the experiment for partial course credit or payment. All had normal or corrected to normal vision, and no one reported any neurological or psychiatric diseases. Five participants were left-handed. The study was approved by the United Ethical Review Committee for Research in Psychology (Hungary), and was carried out in accordance with the Declaration of Helsinki. Written informed consent was obtained from all participants.

\section{Stimuli and procedure}

The experimental stimuli (summarized in Fig. 1 along with the experimental procedure) included both task-relevant and task-irrelevant stimuli presented on an 18" CRT monitor (LG Flatron 915FT Plus, $75 \mathrm{~Hz}$ refresh rate, $1024 \times 768$ px screen resolution) at a viewing distance of $140 \mathrm{~cm}$.

The purpose of the task-relevant stimuli (a tracking task) was to engage the participants' attention. A red dot (RGB $0.6,0,0)$ with a diameter of $0.14^{\circ}$ of visual angle served as a fixation point in the center of the screen. A green disc $\left(0.29^{\circ}, \mathrm{RGB} 0,0.5,0\right)$ moved in a pseudorandom fashion around the fixation point. The participant's task was to keep the green disc as close as possible to the fixation point by controlling its movement with the right and left arrow keys of a keyboard. Each time the distance between the green disc and the fixation point exceeded $0.73^{\circ}$ in either direction, the color of the disc changed to blue (RGB $0,0,1)$ to provide visual feedback that the disc was in the "error zone", and this was counted as an error. As long as the disc was in the "error zone", it remained blue. Performance was measured with: (1) number of errors and (2) percentage of time the disc spent in the "error zone" during a sequence.

The purpose of the task-irrelevant stimuli was to elicit vMMN. Eight identical black oblique bars or line drawings (complex shapes) were arranged in a pattern (a $3 \times 3$ grid) around the tracking task. The vertical distance from the fixation point to a bar or a complex shape of the pattern was $2.88^{\circ}$ of visual angle when it was situated above or below the fixation point, and the horizontal distance was $2.92^{\circ}$ when it was on either side (see Fig. 1a). Each bar stimulus was $0.12^{\circ}$ of visual angle wide and $1.74^{\circ}$ long. Each complex shape was $2.19^{\circ}$ of visual angle at its larger dimension. Stimuli were presented as dark objects $\left(0.14 \mathrm{~cd} / \mathrm{m}^{2}, \mathrm{RGB} 0,0,0\right)$ on a gray background $\left(15.58 \mathrm{~cd} / \mathrm{m}^{2}, \mathrm{RGB} 0.5,0.5,0.5\right)$.

The experiment included the following four conditions: an oddball condition with an oblique bar pattern as the 
Fig. 1 Experimental paradigm and stimuli. a Stimuli in each condition. b Experimental procedure. $\mathbf{c}$ The tracking task area in the case of successful and unsuccessful tracking a

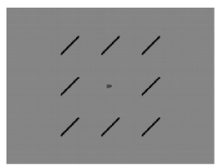

$45^{\circ}-225^{\circ}$

standard

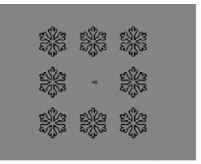

snowflake

standard

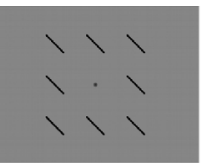

$135^{\circ}-315^{\circ}$

control

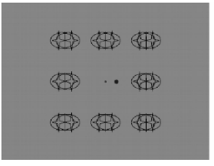

abstract control

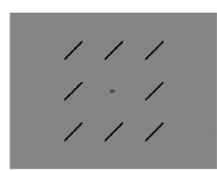

$45^{\circ}-225^{\circ}$

standard
Deviant Snowflake Oddball

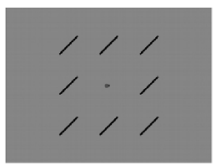

$45^{\circ}-225^{\circ}$ standard

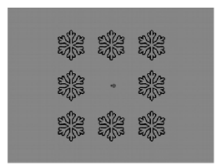

snowflake deviant

Deviant Bar Oddball

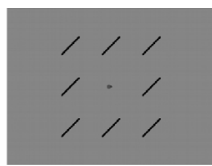

$45^{\circ}-225^{\circ}$

deviant

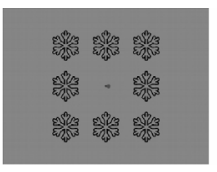

snowflake standard

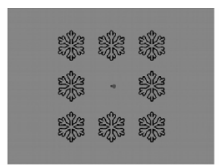

snowflake standard

Equiprobable Bar

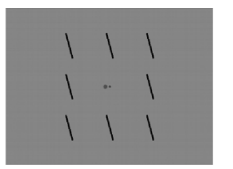

$105^{\circ}-285^{\circ}$

control

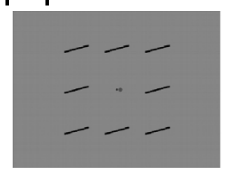

$15^{\circ}-195^{\circ}$

control

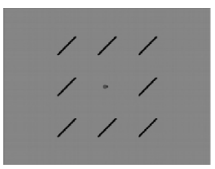

$45^{\circ}-225^{\circ}$

control

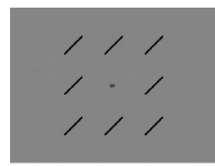

$45^{\circ}-225^{\circ}$

standard

Equiprobable Shape

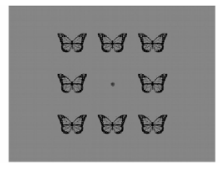

butterfly

control

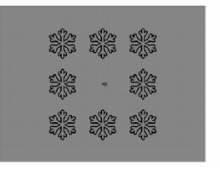

snowflake

control

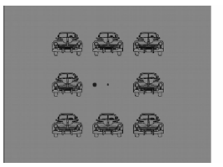

car control

C

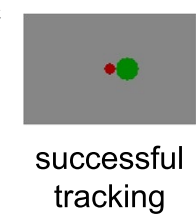

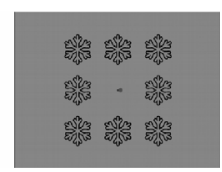

snowflake standard
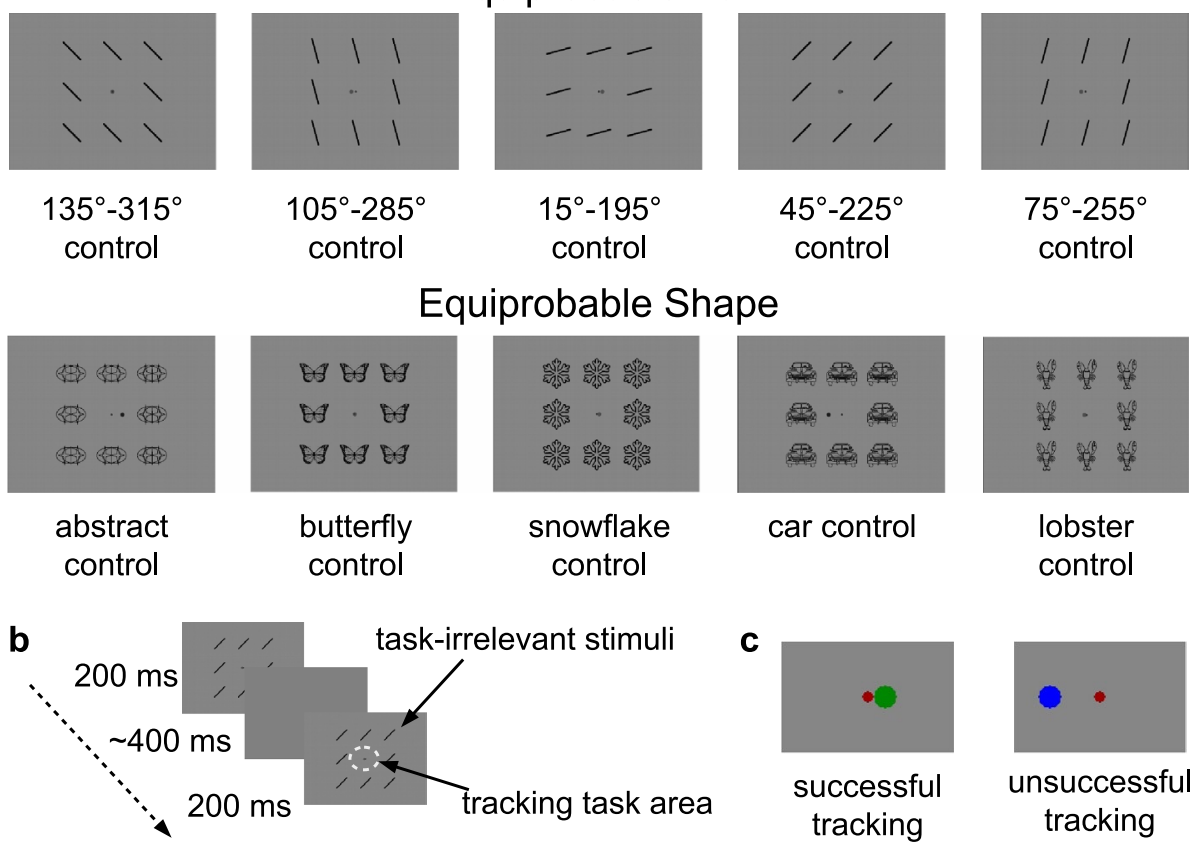

$75^{\circ}-255^{\circ}$

control

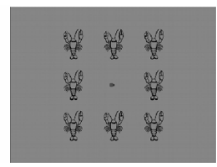

lobster

control

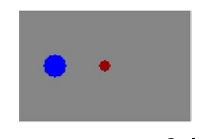

unsuccessful tracking

standard and a snowflake pattern as the deviant stimuli (Deviant Snowflake Oddball); an oddball condition with a snowflake pattern as the standard and an oblique bar pattern as the deviant stimuli (Deviant Bar Oddball); an equiprobable condition with oblique bar patterns of five different orientations as control stimuli (Equiprobable Bar); an equiprobable condition with five different shape patterns as control stimuli (Equiprobable Shape). In the oddball conditions the bars had a $45^{\circ}-225^{\circ}$ orientation. In the Equiprobable Bar condition bars with orientations of $15^{\circ}-195^{\circ}, 75^{\circ}-255^{\circ}, 105^{\circ}-285^{\circ}$ and $135^{\circ}-315^{\circ}$ were added (i.e., the orientations were equally distributed within the $360^{\circ}$ ). In the Equiprobable Shape condition the additional shapes were a butterfly, a bilateral abstract pattern, the front view of a car, and a lobster. The only criterion for choosing the complex shapes was there being no obvious systematic difference between them.

Each condition had a total of 600 stimuli divided into three sequences of 200 stimuli for a total of 12 sequences. For the oddball conditions the probability was 0.8 for the standard and 0.2 for the deviant (480 standard and 120 deviant stimuli in each condition, 160 standard and 40 deviant stimuli in each sequence). In the equiprobable conditions each stimulus had a 0.2 probability, matching the deviant's probability from the oddball conditions (120 stimuli of each overall, 40 of each within a sequence). The presentation order of the sequences was randomized for each participant.

The stimulus presentation time was $200 \mathrm{~ms}$; the average inter-stimulus-interval (ISI) was $400 \mathrm{~ms}$ (range 360-440 ms in $13.33 \mathrm{~ms}$ steps). In the oddball conditions there were 
Table 1 a Conditions and stimuli. b Calculations for the difference waves (vMMN, SSA, vMMN and SSA). c Regions included in the analysis, each region was analyzed separately. $d$ Factors of the univariate analysis of variance

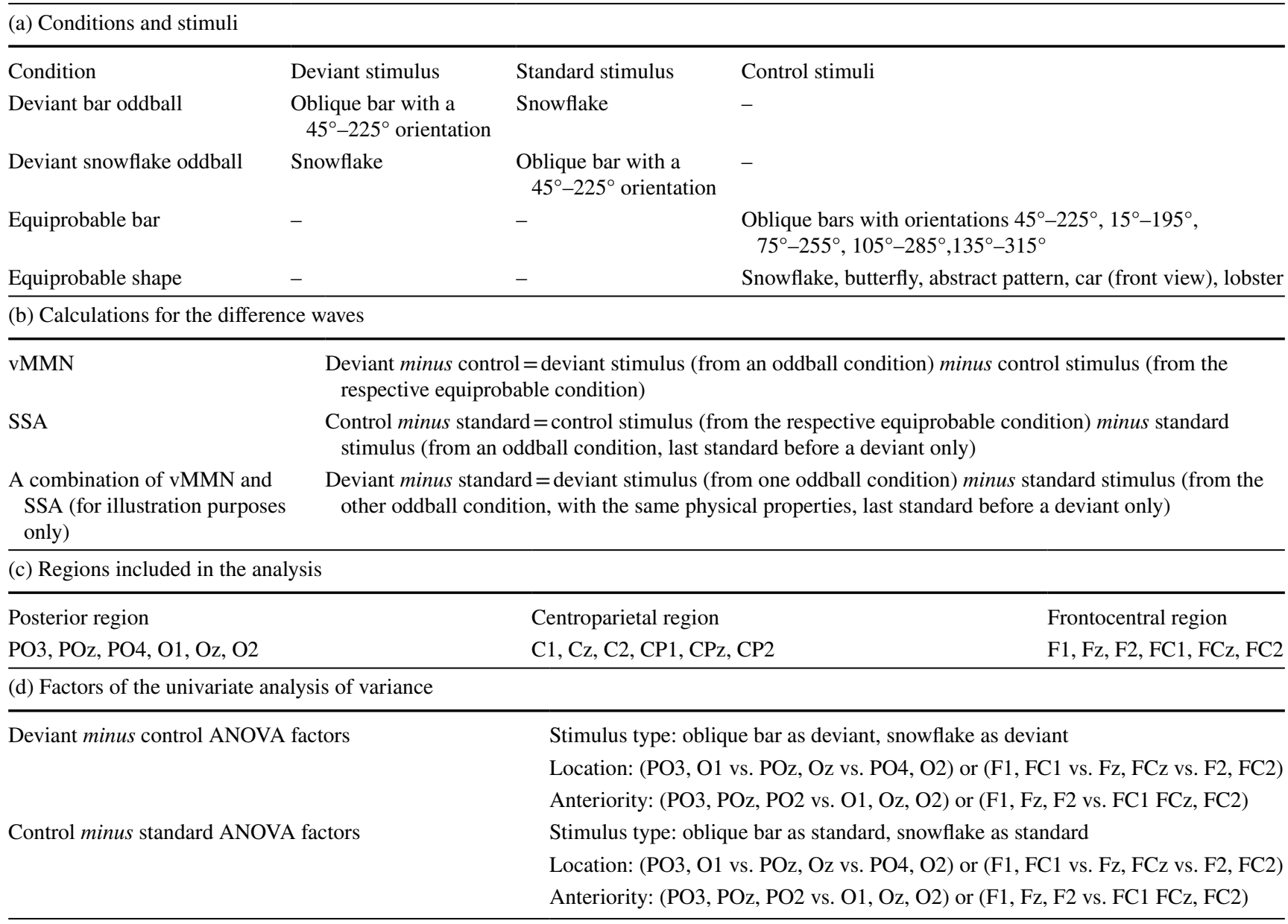

between 2 and 6 standard stimuli between two deviants, whereas in the equiprobable conditions the stimuli were randomized with no two identical stimuli occurring in succession.

The participant sat in a dark room in a comfortable chair with a keyboard on his/her lap in front of the screen on which the stimuli were presented. The experiment started with a 3-min practice during which only the tracking task was presented, and the participant was instructed to keep the green disc as close to the fixation point as possible. EEG was not recorded for the practice.

The experiment started after the practice. The participant was told to focus on the tracking task and ignore the distracting stimuli. After each sequence there was a short break, and the participant started the next sequence with a key press. Each sequence lasted for approximately $2 \mathrm{~min}$. The number of errors was displayed on the screen after each sequence. The duration of the experimental part of the session was approximately $1 \mathrm{~h}$.

A summary of the conditions and the stimuli can be found in Table 1a.
This experiment was realized using Cogent 2000 [within MATLAB (MathWorks, Inc. 2015)] developed by the Cogent 2000 team [Wellcome Department of Imaging Neuroscience (Cogent, http://www.vislab.ucl.ac.uk/Cogent/)].

\section{Recording and measuring electric brain activity}

Brain electric activity was recorded [bandwidth: DC- $100 \mathrm{~Hz}$; sampling rate $500 \mathrm{~Hz}$; Synamps 2 amplifier, NeuroScan recording system (Compumedics Ltd)] with $\mathrm{Ag} / \mathrm{AgCl}$ electrodes placed at 61 locations according to the extended 10-20 system using an elastic electrode cap (EasyCap, Brain Products $\mathrm{GmbH}$ ). The reference electrode was on the nose tip, and the data were offline re-referenced to the average activity. Eye movements were recorded with four electrodes placed around the eyes. Horizontal EOG was recorded with a bipolar configuration between electrodes positioned lateral to the outer canthi of the eyes (one electrode on each side). Vertical eye movement was monitored with a bipolar montage between two electrodes, one placed above and one below the left eye. The impedance of all electrodes was kept 
below $10 \mathrm{k} \Omega$. The EEG signal was band-pass-filtered offline with a non-causal Kaiser-windowed FIR filter (lowpass filter parameters: cutoff frequency of $30 \mathrm{~Hz}$, beta of 12.2653, a transition band of $10 \mathrm{~Hz}$; highpass filter parameters: cutoff frequency of $0.1 \mathrm{~Hz}$, beta of 5.6533, a transition band of $0.2 \mathrm{~Hz}$ ). Epochs with a duration of $600 \mathrm{~ms}$, including a $100 \mathrm{~ms}$ pre-stimulus interval, were extracted for each event and averaged for each type of stimuli (see Data Analysis). The mean voltage during the $100 \mathrm{~ms}$ pre-stimulus interval served as the baseline for amplitude measurements, and epochs with an amplitude change exceeding $100 \mu \mathrm{V}$ on any channel were excluded from further analysis.

\section{Data analysis}

Performance on the tracking task was compared across conditions to ensure that participants attended to the task equally. The number of errors and percentage of time spent in the "error zone" were recorded for each sequence, averaged per condition for each participant, and then compared with a one-way non-parametric analysis of variance test (Friedman test) with the factor Condition (four levels: Deviant Snowflake Oddball, Deviant Bar Oddball, Equiprobable Bar, Equiprobable Shape).

Epochs were averaged for the following types of stimuli: (1) deviant for each oddball condition, (2) last standard immediately before a deviant for each oddball condition, and (3) the respective control stimuli (the $45^{\circ}-225^{\circ}$ bar and the snowflake) from each equiprobable condition. The number of averaged epochs for each of the six stimulus types was between 111 and 113 per participant; $5.70-6.89 \%$ of the epochs were rejected.

The analyses focused primarily on ERPs measured at the electrode sites over the posterior areas $(\mathrm{PO} 3, \mathrm{POz}, \mathrm{PO} 4$, $\mathrm{O} 1, \mathrm{Oz}$, and $\mathrm{O} 2$ ) within the 120-350 ms window, i.e., the expected locations and time window of vMNN as our main goal was to identify the presence of vMMN for stimuli from different categories. Because it was possible that the categorical difference also resulted in a difference elsewhere, two additional regions were also examined: a centroparietal (CP1, CPz, CP2, C1, Cz, C2) and a frontocentral (FC1, $\mathrm{FCz}, \mathrm{FC} 2, \mathrm{~F} 1, \mathrm{Fz}, \mathrm{F} 2$ ) region. The choice of both additional regions was based on visual inspection of the ERPs and the scalp distributions to the difference waves.

To identify changes related to vMMN, the ERPs elicited by the control stimuli with the same physical properties in the equiprobable conditions were subtracted from the ERPs to the deviant stimuli (deviant minus control) (see Kimura et al. 2010). To assess the involvement of SSA, the ERP difference of the standard stimuli and the control stimuli sharing the same physical properties was also calculated (control minus standard). To illustrate the difference between the vMMN and the vMMN plus SSA, we present the deviant minus standard difference. This difference was calculated from the standard and deviant stimuli with identical physical properties, e.g., the ERP to the standard stimuli from the Deviant Bar Oddball condition was subtracted from the ERP to the deviant stimuli from the Deviant Snowflake Oddball condition (both were snowflake patterns).

One-sample $t$ tests were run on the difference potentials to identify consecutive significant deviations from zero in the negative direction. Only significant deviations for at least 11 consecutive data points $(20 \mathrm{~ms})$ at all six electrode sites in a region were considered to indicate the presence of vMMN in the relevant condition for the deviant minus control difference potential (cf. Guthrie and Buchwald 1991). Peak and onset latencies were measured to assess the time of detecting the deviancy. Peak latencies were measured with a sliding window algorithm. As a first step, the algorithm finds the local maximum of the ERP. Then the algorithm examines whether it is a peak (i.e., the value is larger than both flanking values within the window) or a maximum (i.e., the value is the largest within the window, but it is also at the edge of the window). In the latter case, the window slides toward the maximum with the size of a half-window. The size of the sliding window was ten data points. The onset latency of the difference potentials was defined as the latency of the midpoint between the baseline and the peak amplitude and was calculated from the grand average with the jackknifebased method described by Miller et al. (1998), see also Luck (2005); Ulrich and Miller (2001). Amplitudes and integrated activity were measured to assess the magnitude of vMMN. Amplitude values were calculated as the average of the $\pm 10 \mathrm{~ms}$ range (a $20 \mathrm{~ms}$ duration) around the largest negativity in the grand average. Finally, the integrated activity (i.e., the magnitude of the activity as whole) was calculated as the sum of the amplitude values within the range for which the point-by-point differences between the deviant and control stimuli were statistically significant. In all cases, three-way univariate ANOVAs were calculated for each region with factors Stimulus Type (oblique bar pattern as deviant, snowflake pattern as deviant), Location (left, center, right), and Anteriority (posterior, anterior). Similar calculations were carried out for the control minus standard differences to determine the magnitude of SSA. When necessary, Greenhouse-Geisser corrections were used. For post hoc comparison the Tukey HSD test was applied. Only the main effects and interactions for Stimulus Type are reported in the text, but a summary of all results can be found in Supplementary Information 1. A summary of the performed calculations can be found in Table $1 \mathrm{~b}-\mathrm{d}$.

The EEG data were processed with MATLAB R2015a (MathWorks, Inc. 2015) and the EEGLAB 13.6.5b toolbox (Delorme and Makeig 2004). Statistical analyses were performed with Statistica v13 (Statsoft, Inc. 2016). 


\section{Results}

\section{Behavioral results}

The mean number of errors was: $0.75(\mathrm{SD}=0.88)$ for the Deviant Snowflake Oddball condition, 1.0 $(\mathrm{SD}=1.08)$ for the Deviant Bar Oddball condition, $0.93(\mathrm{SD}=0.86)$ for the Equiprobable Bar condition, and $0.54(\mathrm{SD}=0.49)$ for the Equiprobable Shape condition, i.e., the green disc went to the "error zone" less than once per sequence on average. There was no significant difference between the conditions (Friedman test, $\chi^{2}(3, N=19)=3.8, p=0.284$ ). The mean percentage of time spent in the "error zone" was: $0.16 \%(\mathrm{SD}=0.26 \%)$ for the Deviant Snowflake Oddball, $0.17 \%(\mathrm{SD}=0.24 \%)$ for the Deviant Bar Oddball, $0.19 \%(\mathrm{SD}=0.24 \%)$ for the Equiprobable Bar, and $0.08 \%$ $(\mathrm{SD}=0.07 \%)$ for the Equiprobable Shape. There was no significant difference between the conditions (Friedman test, $\left.\chi^{2}(3, N=19)=1.88, p=0.597\right)$. This high performance ${ }^{1}$ suggests that participants attended to the task.

\section{Event-related potentials}

As Fig. 2 shows, the ERPs to the oblique bar and the snowflake patterns were dominated by the P2 component within the 200-300 ms range at the posterior sites. A discernable N1 can be observed only for the oblique bar pattern as deviant. As a marked difference between the ERPs, only the snowflake pattern elicited offset components [a negative-positive response in the 100-200 ms range after stimulus offset (offset was at $200 \mathrm{~ms}$ after onset)]. The patterns were reversed, and the ERPs were smaller at the frontocentral sites. The morphology of the ERPs in the equiprobable sequences was similar to the respective ERPs from the oddball sequences, and visual inspection shows them to be between the ERPs to the respective deviant and standard stimuli in the vicinity of N1. The ERPs to the stimuli in the equiprobable conditions are depicted in Supplementary Information 2.

\footnotetext{
1 There is no absolute measure of performance to which to compare the participants' results in this task. Consequently, performance can only be compared between conditions. However, it is possible to obtain number of errors and time spent in the "error zone" for the length of an experimental sequence if the task is left to run without human interference. The task was run 96 times with the length of each run being equal to one experimental sequence ( $2 \mathrm{~min}$ ), which averaged 11.45 errors and $53.39 \%$ of time spent in the "error zone" per sequence. This would be the performance if the participant does not do the task at all.
}

The deviant minus standard, ${ }^{2}$ deviant minus control and control minus standard difference potentials are depicted in Fig. 3a-c show the ranges for the integrated activity (the duration of consecutive data points for which the difference significantly deviated from 0 in the negative direction) around the peak latency in the grand average for the deviant minus control and control minus deviant difference potentials, respectively. Scalp distributions for the average of the $20 \mathrm{~ms}$ range around the peak latency in the grand average at $\mathrm{Oz}$ for each difference are also depicted.

According to the criterion [11 consecutive data points (20 ms) of significant deviation from 0], both the deviant oblique bars and the deviant snowflakes elicited vMMN at the posterior and frontocentral, but not at the centroparietal locations (Fig. 3b). Thus, our analysis was limited to those two regions. The narrowest range for the oblique bar pattern was $48 \mathrm{~ms}$ at $\mathrm{PO} 3$ for the posterior region and $32 \mathrm{~ms}$ at $\mathrm{FC} 1$ for the frontocentral region, and for the snowflake pattern it was $56 \mathrm{~ms}$ at $\mathrm{Oz}$ for the posterior region and $26 \mathrm{~ms}$ at $\mathrm{FCz}$ for the frontocentral region. ${ }^{3}$

Table 2 shows the average peak and onset latency values of the deviant minus control and the control minus standard difference potentials, the average peak amplitude values of these difference potentials, and the averaged integrated activity for the highlighted ranges.

According to a three-way univariate ANOVA of the peak latencies of the deviant minus control difference potentials for the posterior region with factors of Stimulus Type (oblique bar pattern as deviant, snowflake pattern as deviant), Location (PO3 and $\mathrm{O} 1, \mathrm{POz}$ and $\mathrm{Oz}, \mathrm{PO} 4$ and $\mathrm{O} 2$ ), and Anteriority (PO4, POz, $\mathrm{PO} 3$ vs. O1, Oz, O2), the main effect of Stimulus Type was significant, $F(1,18)=9.16, p=0.007$, $\eta_{\mathrm{p}}{ }^{2}=0.337$ ), and the latency of vMMN was longer for the deviant snowflake pattern. In an ANOVA with the same factors for the onset latencies, the vMMN latency remained longer for the snowflake pattern as deviant, but the difference did not reach significance, $F(1,18)=4.06, p=0.059$,

\footnotetext{
${ }^{2}$ Deviant minus standard differences were calculated as the difference between the oblique bar pattern as a deviant minus the oblique bar as a standard, and the snowflake pattern as a deviant minus the snowflake pattern as a standard. These differences involve both deviant-related and stimulus-specific adaptation effects; therefore we present these differences only as an illustration.

3 The range at FC1 for the snowflake pattern was $10 \mathrm{~ms}$; however, this site was included for the sake of the statistical analysis. For both conditions there is a monotonic linear increase from FC1 to FC2, although the slope for the oblique bar pattern is steeper than the slope for the snowflake pattern, and the small value at FC1 likely reflects the continuation of the linear trend as well as a distribution slightly to the right for vMMN at the FC electrode sites. The latter was also the reason for our decision to include the frontocentral region in the case of the snowflake pattern as a deviant, even though there was one electrode site (FC1) at which the range of consecutive data points significantly different from 0 did not reach $20 \mathrm{~ms}$.
} 
a
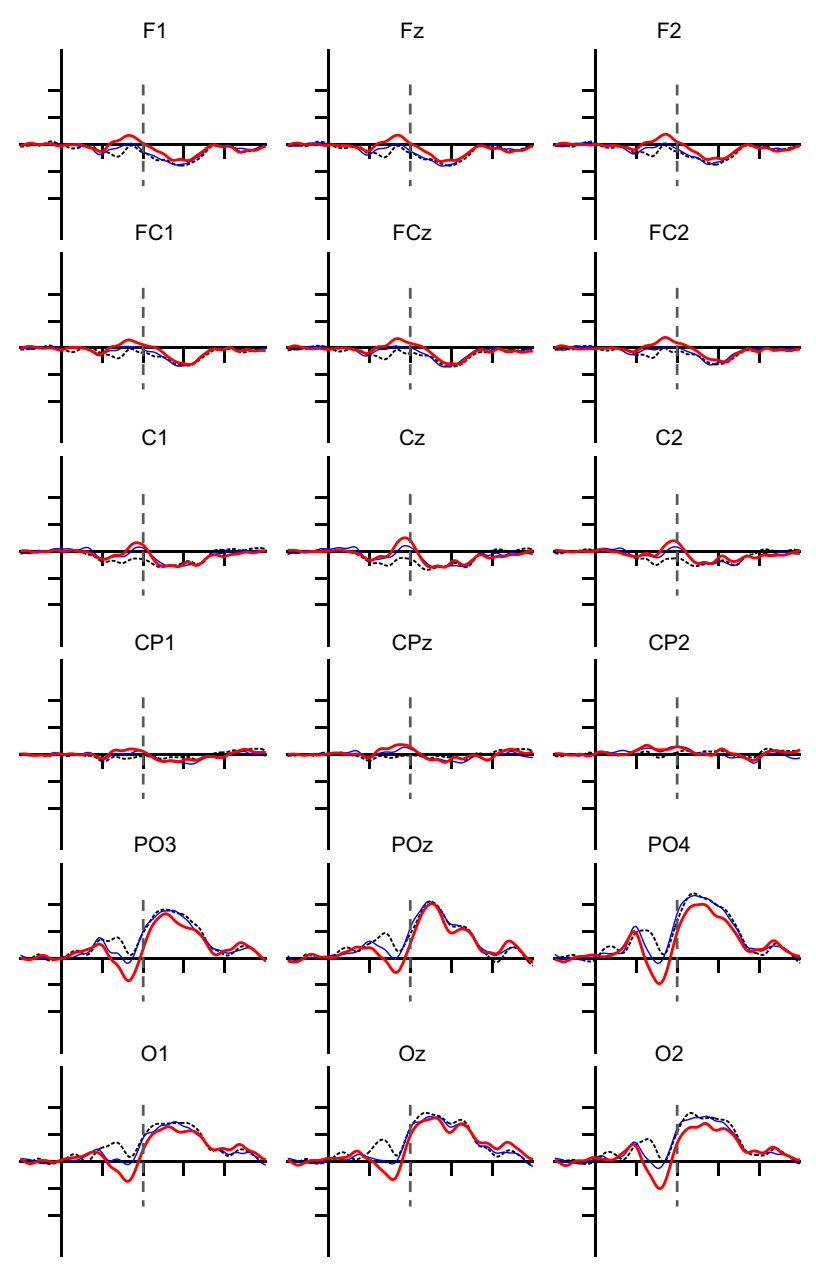

$\mathrm{O} 2$

$$
\text { - deviant } \quad \text { control }
$$

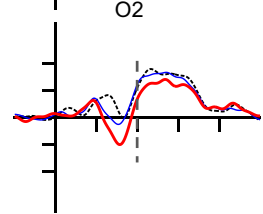

b
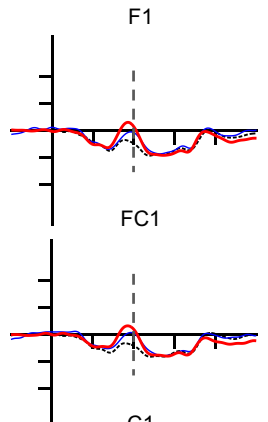

C1
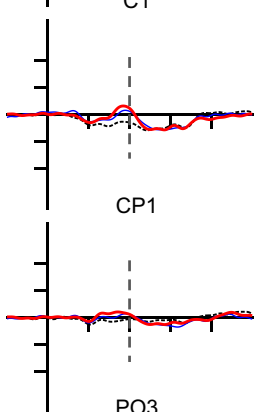

snowflake
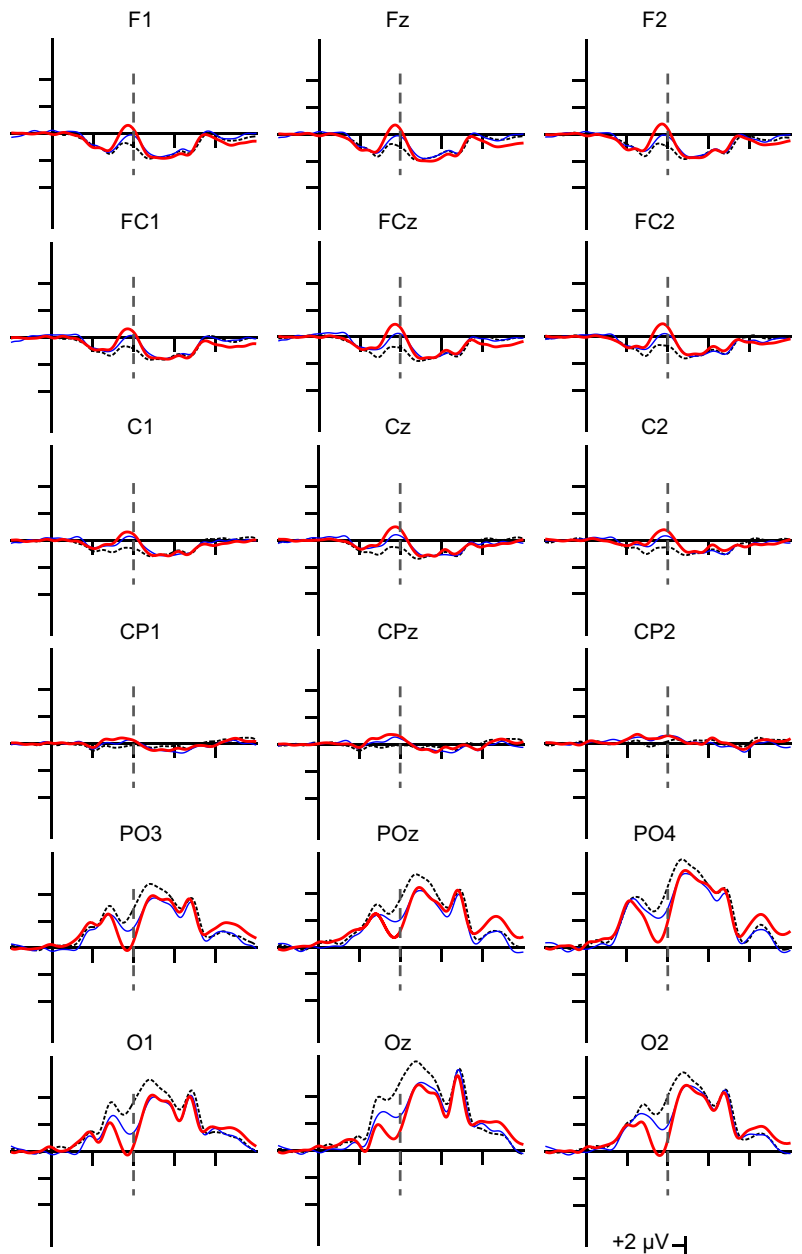

---- standard

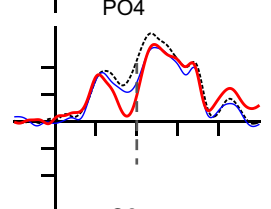

Fig. 2 ERPs of the standard, deviant, and control stimuli for the oblique bar pattern (a) and the snowflake pattern (b) registered at posterior channels, centroparietal and frontocentral sites

$F$ and $p$ corrected according to the jackknife-based method. In the ANOVA on the peak amplitude values there was no significant main effect of Stimulus Type. Finally, as an additional measure for the robustness of vMMN, the integrated activity was compared with the same ANOVA and factors. The interaction between Stimulus Type and Anteriority was significant, $F(1,18)=13.99, p=0.002, \eta_{\mathrm{p}}{ }^{2}=0.437$. Post hoc tests revealed that the vMMN activity was larger for the oblique bar at the PO sites $(p=0.019)$.

The same analyses were performed for the frontocentral region with factors of Stimulus Type (oblique bar pattern as deviant, snowflake pattern as deviant), Location (FC1 and $\mathrm{F} 1, \mathrm{FCz}$ and $\mathrm{Fz}, \mathrm{FC} 2$ and $\mathrm{F} 2$ ), and Anteriority (FC1, FCz, $\mathrm{FC} 2$ vs. $\mathrm{F} 1, \mathrm{Fz}, \mathrm{F} 2)$. For peak latencies there was the interaction between Anteriority and Stimulus Type, $F(1,18)=5.69$, $p=0.028, \eta_{\mathrm{p}}^{2}=0.240$. The latency for the oblique bar pattern as deviant was shorter only at the F sites and was shorter than the latency for the same at the FC sites, $p$ s between 0.003 and 0.025 . There was no difference in onset latencies or peak amplitudes. Concerning the vMMN activity, the only significant interaction including Stimulus Type was the Anteriority $\times$ Stimulus Type interaction, $F(1,18)=5.73$, $p=0.028, \eta_{\mathrm{p}}^{2}=0.242$ with the activity being larger for the oblique bar pattern as deviant at the FC sites than at F sites for both deviants.

Stimulus-specific adaptation effects were assessed with the control minus standard difference potentials. SSA was clearly visible in the posterior and frontocentral regions with the narrowest range being $40 \mathrm{~ms}$ for the oblique bar pattern as standard at POz for the posterior region and $28 \mathrm{~ms}$ at F2 for the frontocentral region, and $98 \mathrm{~ms}$ at $\mathrm{O} 2$ in the posterior region and $58 \mathrm{~ms}$ at $\mathrm{F} 1$ and $\mathrm{Fz}$ in the frontocentral region for the snowflake pattern. The case for the centroparietal region is less straightforward. SSA is clearly present for the 


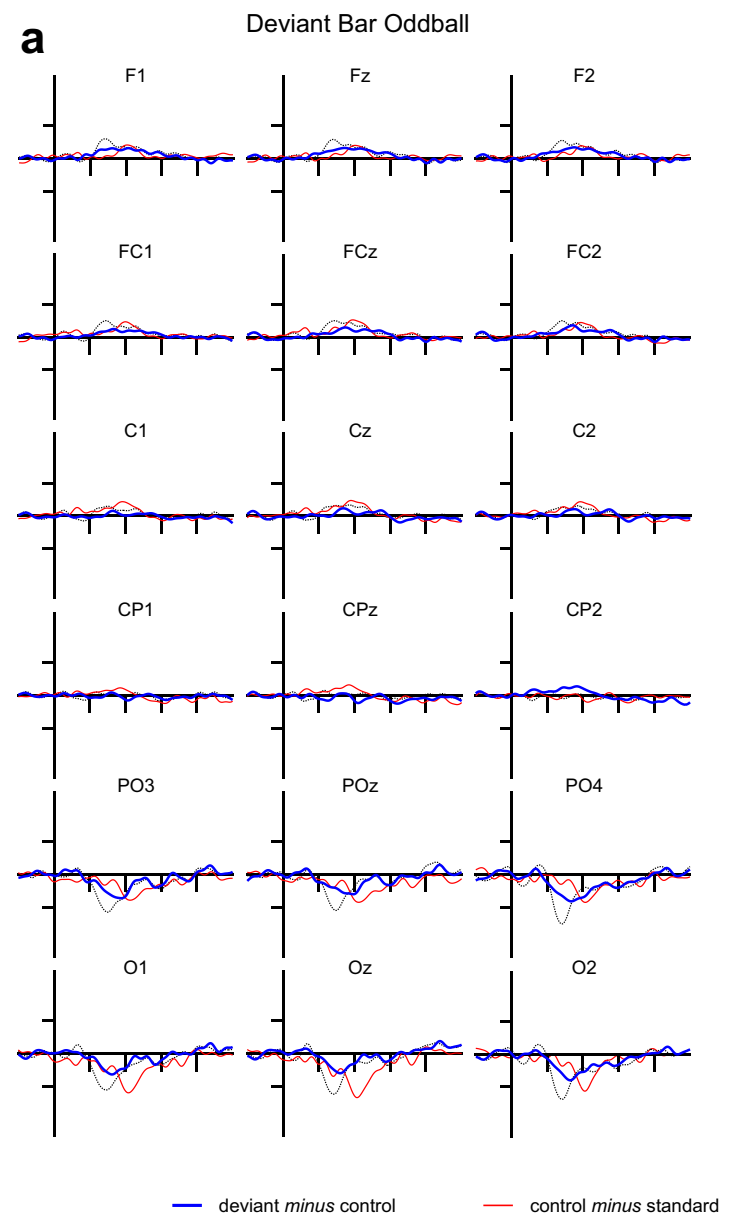

Fig. 3 a The deviant minus control, control minus standard, and deviant minus standard difference potentials for the two oddball conditions registered at posterior, centroparietal and frontocentral sites. b The deviant minus control difference potentials for the oblique bar pattern and the snowflake pattern as deviant stimuli registered at posterior, centroparietal and frontocentral sites the ranges for the integrated activity as well as scalp distributions for the $20 \mathrm{~ms}$ range

snowflake pattern as standard at both $\mathrm{C}$ and $\mathrm{CP}$ sites in the time window of 100-250 ms at the $\mathrm{C}$ sites and 130-200 ms at the $\mathrm{CP}$ sites, whereas there are two time windows at $\mathrm{Cz}$, $\mathrm{C} 2$, and $\mathrm{CPz}$ at approximately $100-150 \mathrm{~ms}$ and $180-230 \mathrm{~ms}$ after stimulus onset, the first time window also being present for $\mathrm{C} 1$. As it is unclear which time window should be used and there are two electrode sites that do not fulfill the criterion of 11 consecutive data points, further statistical analysis was not conducted for this region.

For the posterior region in an univariate three-way ANOVA with the same factors as in the analysis for the deviant minus control difference, the main effect of Stimulus Type was significant, $F(1,18)=238.69, p<0.001$, $\eta_{\mathrm{p}}{ }^{2}=0.930$. Additionally, the Anteriority $\times$ Stimulus Type interaction also reached significance, $F(1,18)=12.30$, $p=0.003, \eta_{\mathrm{p}}{ }^{2}=0.406$. The latency of SSA for the snowflake
Deviant Snowflake Oddball
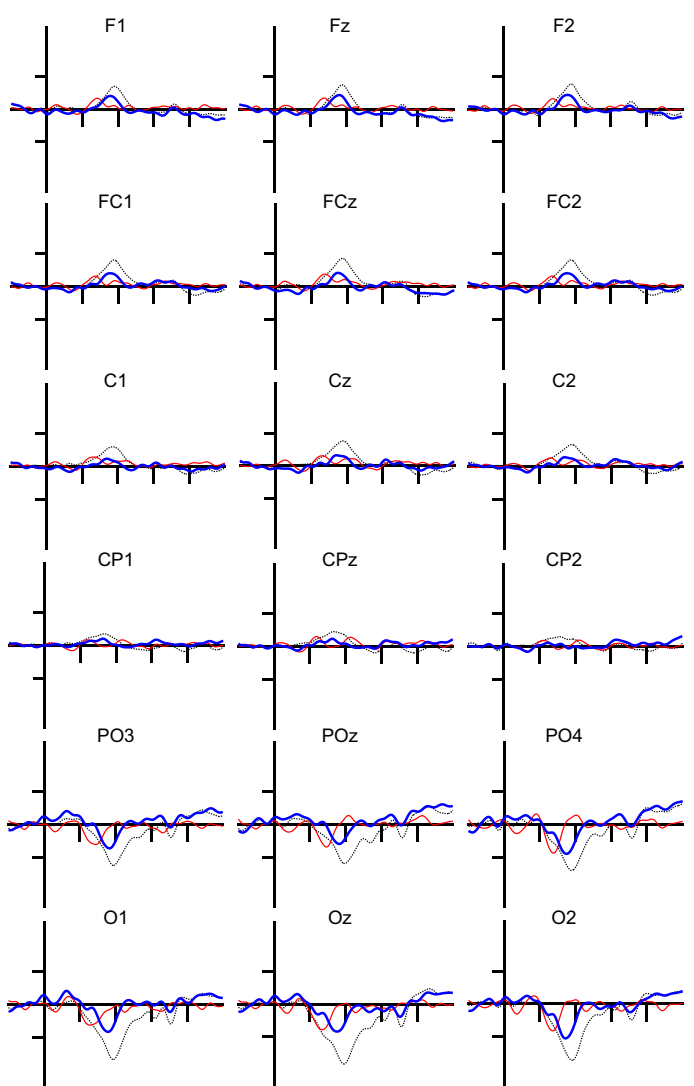

......... deviant minus standard

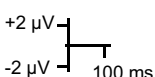

around the peak latency in the grand average at Oz. c The control minus standard difference potentials for the oblique bar pattern and the snowflake pattern as standard stimuli registered at posterior, centroparietal and frontocentral sites the ranges for the integrated activity as well as scalp distributions for the $20 \mathrm{~ms}$ range around the peak latency in the grand average at $\mathrm{Oz}$

pattern as standard was longer overall, and longer at $\mathrm{PO}$ than at $\mathrm{O}$ sites (all $p \mathrm{~s}<0.001)$. The three-way ANOVA for the onset latency confirmed the main effect of Stimulus Type, $F(1,18)=121.23, p<0.001$, respectively (jackknife-based method (Miller et al. 1998; Ulrich and Miller 2001), $F$ and $p$ corrected accordingly). The onset latency was longer for the snowflake pattern as standard. The larger adaptation of the snowflake pattern as standard stimuli is reflected by the larger peak amplitude of the difference potential. According to the ANOVA, the main effect of Stimulus Type was significant, $F(1,18)=4.46, p=0.048, \eta_{\mathrm{p}}{ }^{2}=0.199$. The interaction of Anteriority and Stimulus Type was also significant, $F(1$, $18)=10.95, p=0.004, \eta_{\mathrm{p}}^{2}=0.378$. Post hoc tests showed that the amplitude of SSA for the snowflake pattern as standard was larger only at $\mathrm{O}$ sites $(p s<0.001)$. To illustrate the large adaptation difference, the integrated activities were 
b
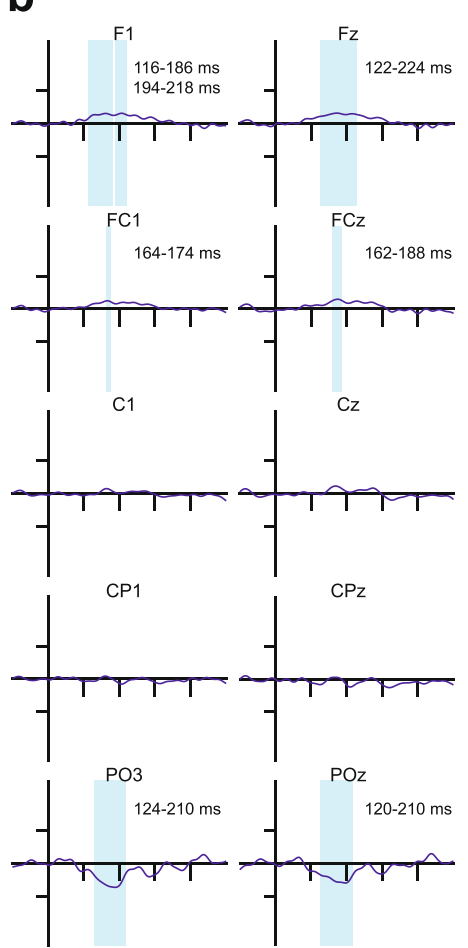

01

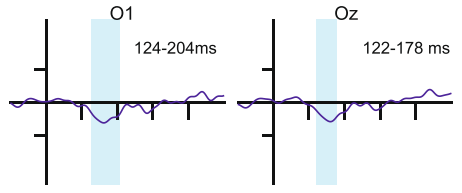

— deviant minus control

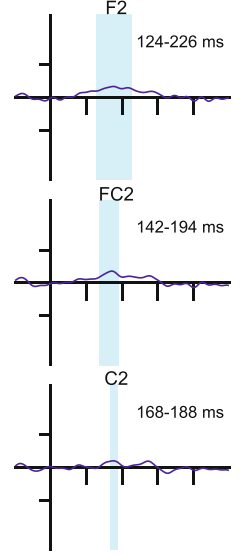

CP2

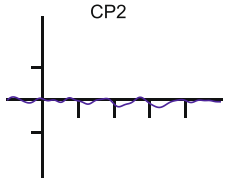

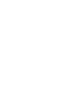
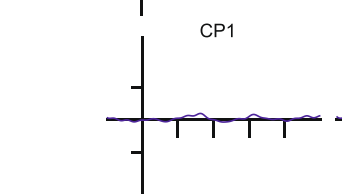

snowflake

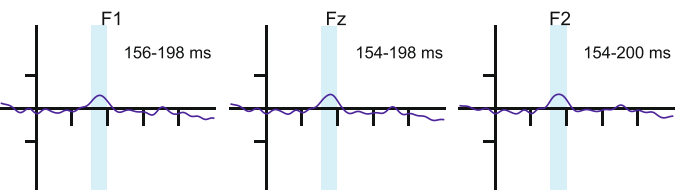

FC1

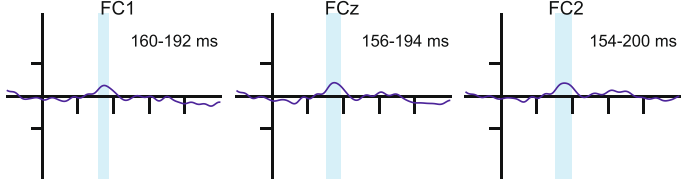

C1

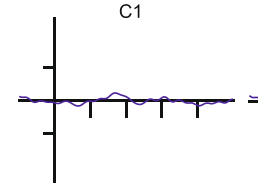

$\mathrm{Cz}$

C2

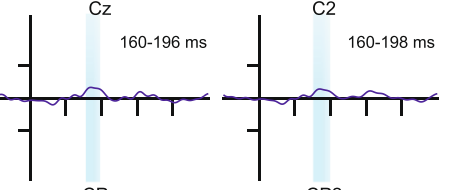

$\mathrm{CPz}$

CP2

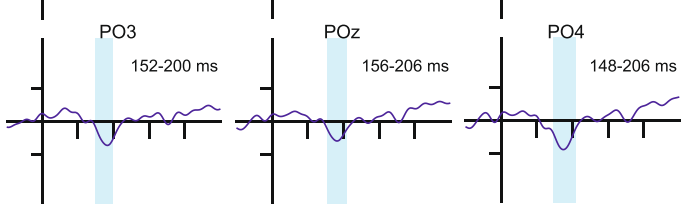

$\mathrm{O} 2$
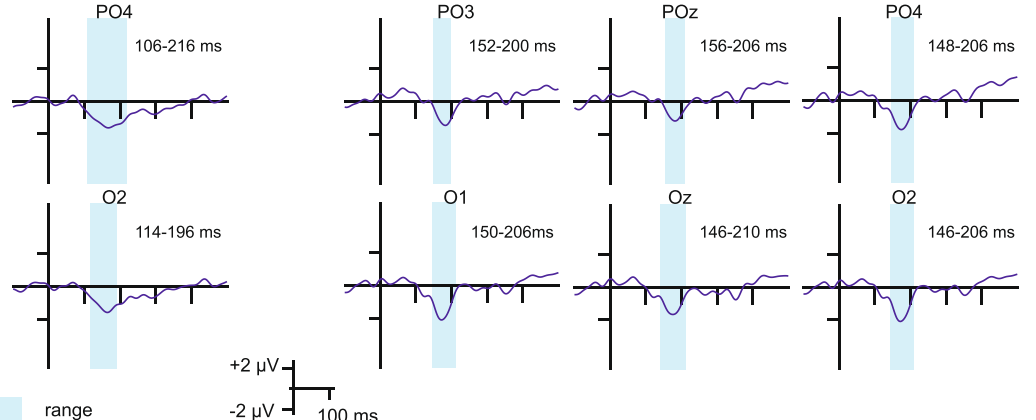

$\mathrm{ms}$

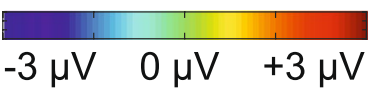

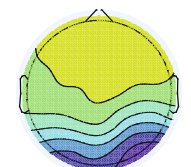

168-188 ms

$150-170 \mathrm{~ms}$

Fig. 3 (continued)

also compared (see Table 2). All main effects and interactions including the factor Stimulus Type were significant: Stimulus Type, $F(1,18)=29.80, p<0.001, \eta_{\mathrm{p}}{ }^{2}=0.623$, Anteriority $\times$ Stimulus Type, $F(1,18)=12.90, p=0.002$, $\eta_{\mathrm{p}}{ }^{2}=0.418$, Stimulus Type $\times$ Site, $F(2,36)=7.12, p=0.003$, $\eta_{\mathrm{p}}{ }^{2}=0.283$, and the triple interaction of Anteriority $\times$ Stimulus Type $\times$ Location, $F(2,36)=4.62, p=0.016, \eta_{\mathrm{p}}{ }^{2}=0.204$. The SSA activity was larger overall for the snowflake pattern as standard, larger for the snowflake at $\mathrm{O} 1, \mathrm{Oz}, \mathrm{PO} 3$ and $\mathrm{POz}$, and larger at $\mathrm{O} 1$ and $\mathrm{Oz}$ than the PO sites $(p s<0.029)$.

The same analyses were performed for the frontocentral region. For peak latencies there was only a main effect of Stimulus Type, $F(1,18)=166.92, p<0.001, \eta_{\mathrm{p}}{ }^{2}=0.903$ with the latency being longer for the snowflake pattern as standard. The result for the onset latency was the same, $F(1,18)=6.57, p=0.019$. There was no difference in peak amplitude at the frontocentral sites; however, for the SSA integrated activity all main effects and interactions including the Stimulus Type with the exception of the Stimulus Type $\times$ Location interaction were significant: Stimulus Type, $F(1$, $18)=10.76, p=0.004, \eta_{\mathrm{p}}{ }^{2}=0.374$, Anteriority $\times$ Stimulus Type, $F(1,18)=7.25, p=0.015, \eta_{\mathrm{p}}{ }^{2}=0.287$, and the triple interaction of Anteriority $\times$ Stimulus Type $\times$ Location, $F(2$, $36)=5.25, p=0.010, \eta_{\mathrm{p}}{ }^{2}=0.226$. The activity was overall larger for the snowflake pattern as standard, larger at $\mathrm{Oz}$ than $\mathrm{O} 1$ and $\mathrm{O} 2$, and at $\mathrm{Oz}$ and $\mathrm{O} 2$ than at $\mathrm{POz}$ and $\mathrm{PO} 4$.

All results from the statistical analyses can be found in Supplementary Information 1. An additional analysis of the 


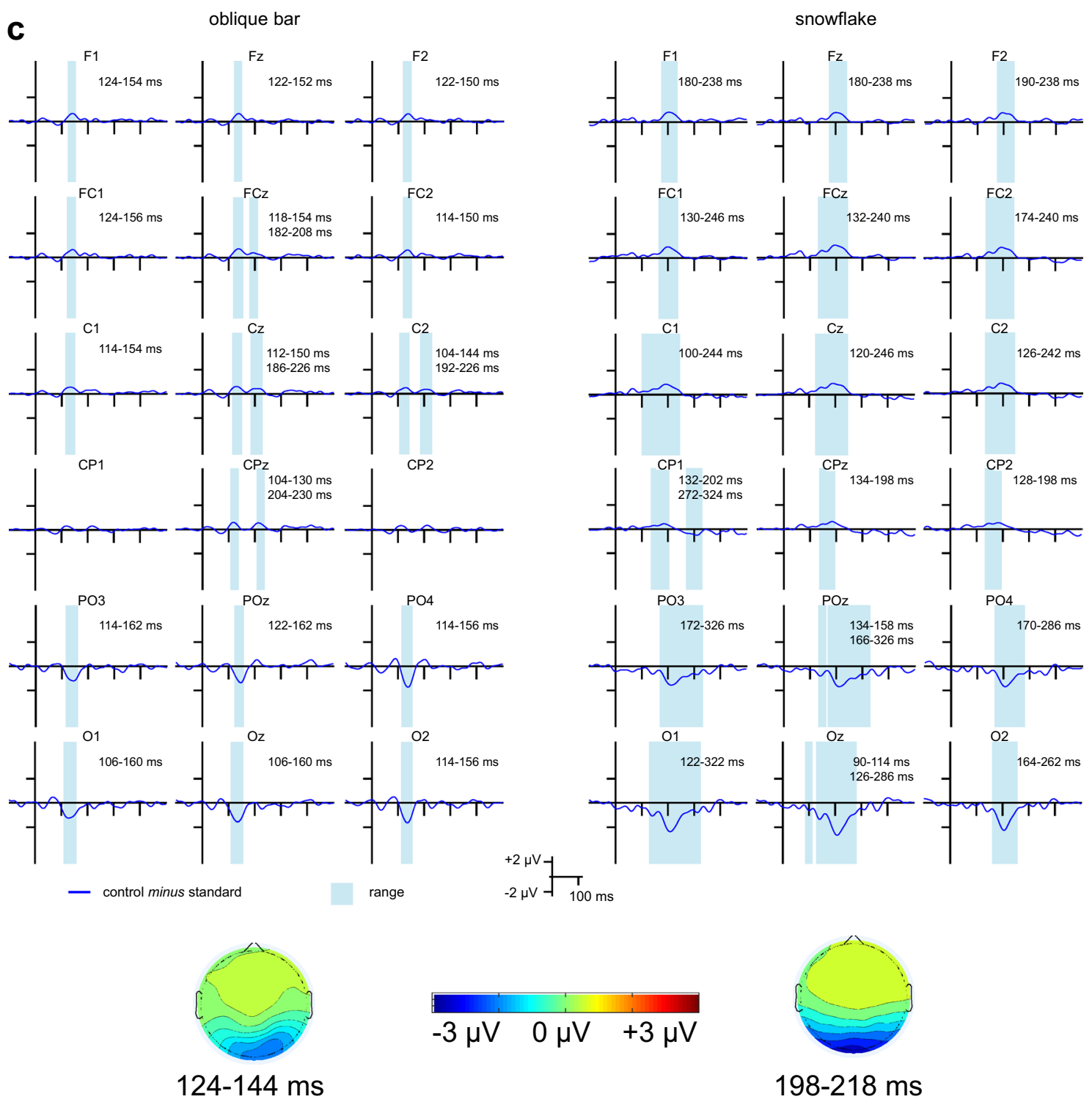

Fig. 3 (continued)

time course of the adaptation of the standard is briefly summarized in Supplementary Information 3. An analysis of the differences in scalp distributions between conditions for the deviant minus control and the control minus standard differences based on the method described by Karniski, Blair, and Snider (1994) is briefly described in Supplementary Information 4.

\section{Discussion}

For both the oblique bar pattern and the snowflake pattern vMMN emerged at the posterior region. Both the peak latency and the onset latency of vMMN were shorter for the oblique bar pattern as deviant compared to the snowflake pattern as deviant (refer to Table 2 for the magnitude of the difference), although for the latter difference only a tendency was observed. The peak vMMN amplitude was less than $-2 \mu \mathrm{V}$ for both patterns, and the difference was not significant. The integrated activity did not differ between the two oddball conditions either. At the frontocentral electrode sites there was a smaller positivity in approximately the same range as the negativity in the posterior region. There were no differences in peak or onset latency between the two deviants as well as no overall difference in either peak amplitudes or integrated activity. The scalp distributions for the deviant minus control difference at the peak latencies did not differ (see Supplementary Information 4). 


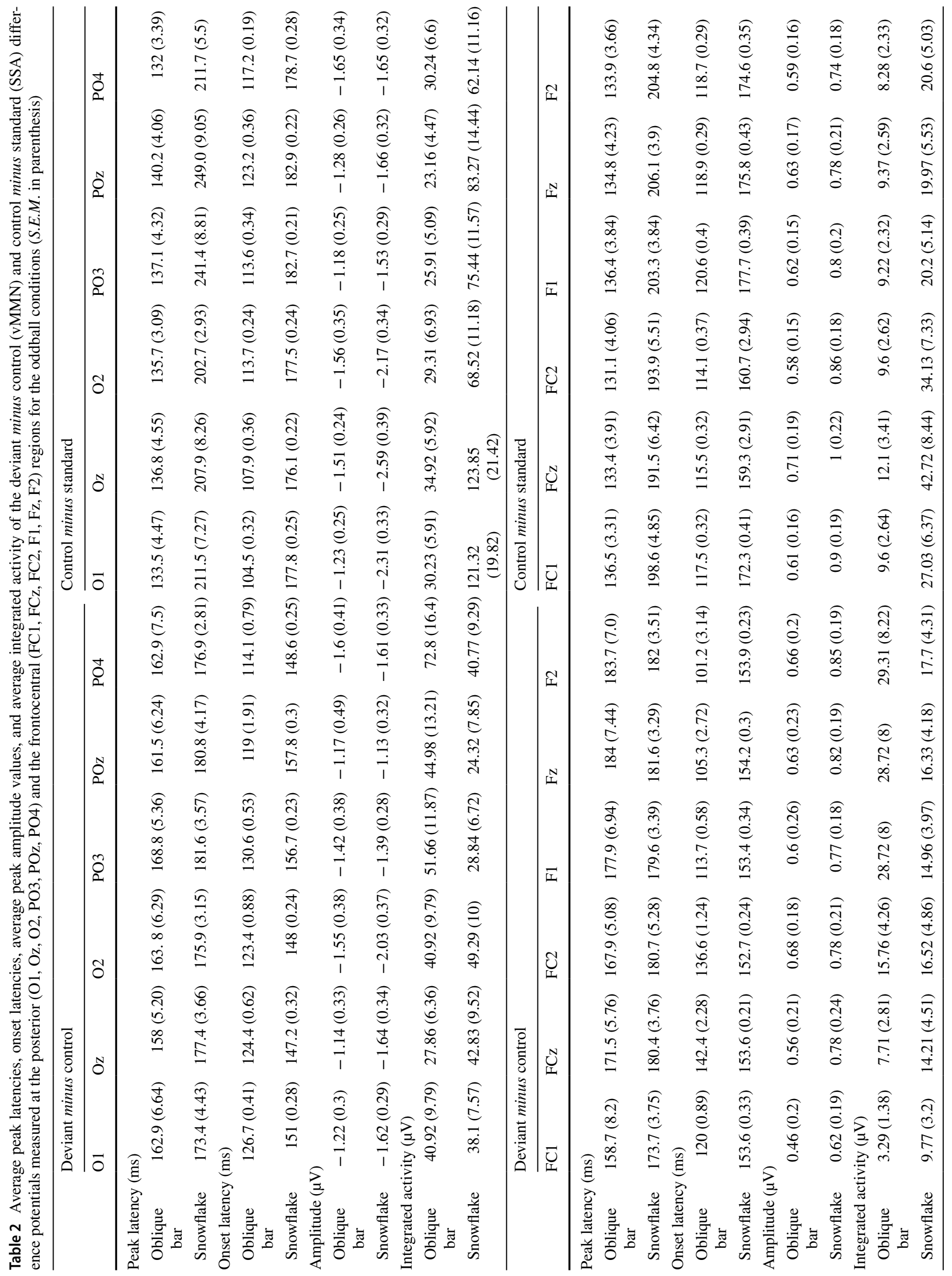


The main purpose of the study was to investigate three issues. First, according to the interpretation of the trace mismatch explanation, vMMN is no more than a sign of the detection of a deviant event. In this respect, the expectation is a similar vMMN to simple and complex deviants. The similarity in magnitude of the deviant minus control differences between the vMMN to the oblique bar pattern and that to the snowflake pattern stimuli corresponds to this interpretation. Second, if the more salient deviant elicits a more robust vMMN, such a result can be attributed to a call for further processing (and possibly to the further processing) of the more complex deviant. The results did not support this possibility. Third, according to the interpretation of the predictive coding account, $\mathrm{vMMN}$ is a correlate of a cascade of adjustment processes between the models of the environment and the representation of incoming stimulation. It is difficult to interpret the emergence of vMMN in the 200-300 ms range to deviants that are unrelated to the standard as a correlate of the adjustment processes. Importantly, we do not claim that the vMMN is not sensitive to such a sequence of processes in cases in which an adjustment between the incoming stimulation and the updating environmental models is a possibility. The present results only show that such a mechanism is not necessary for the emergence of vMMN.

As for the automatically acquired and implicit (non-conscious) memory, it claims that the function of this system is to maintain a representation of the regular characteristics of the environment, and the process underlying the mismatch components is to update the memory system (Winkler and Czigler 1998; Winkler and Schröger 2015). Here, the shorter vMMN latency to the oblique bar pattern (a simple stimulus) indicates that deviancy is detected earlier in the context of complex stimuli than a complex deviant event within the sequence of more simple ones. When the standard and the deviant are similar [e.g., belonging to the same category (oblique lines: Kimura et al. (2009, 2010); facial categories and emotions: Yu et al. (2017), Vogel et al. (2015), Kreegipuu et al. (2013); left vs. right hand: Stefanics and Czigler (2012)], deviant-related negativity included longer latency ranges, or there were mismatch components in various ranges, whereas in the case of highly different standard and deviant (e.g., symmetric vs. asymmetric patterns), the difference potential was confined to an earlier and narrower latency range. Furthermore, using similar standards and deviants such as disappearing parts of an object (Sulykos et al. 2017) and checkerboards with alternating locations of the dark and light squares (Sulykos et al. 2018), an identical earlier phase of the vMMN appeared for both younger and older groups, whereas the later part was absent or diminished in the elderly. This age-related difference can be interpreted as preserved detection of a deviance, but compromised identification of the deviancy.
VMMN for deviant oblique bars has been obtained in former studies using an equiprobable control with withincategory stimuli (i.e., oblique bars with a different orientation) as standards, but the results are equivocal. Astikainen et al. (2008) and Kimura et al. (2009) presented single bars. Astikainen et al. (2008) obtained a deviant minus control difference in the 185-205 ms range. In the study by Kimura et al. (2009) applying an equiprobable control eliminated the difference in the range that corresponded to a posterior negativity (N1), but vMMN emerged at a later latency range (200-250 ms). The results of Kimura et al. (2010) were similar. Kimura and Takeda (2013) used oblique bar patterns as stimuli, similar to the present study, and as in their earlier studies, the control eliminated the early part of the response, but preserved the later difference. Finally, File et al. (2017) presented a texture consisting of oblique bars. In this study the control procedure fully eliminated the deviance effect. It seems that a complex interaction occurs between taskrelated attentional demand [auditory task: Astikainen et al. (2008), identification of a feature of the vMMN-related stimuli: Kimura et al. (2009, 2010); visual discrimination: Kimura and Takeda (2013); video games: File et al. (2017)] and the stimulus characteristics (single bar, bars around the task-field, texture of bars in non-attended part of the visual field). Early vMMN effects can be considered as a detection of change, and later effects as an identification of deviancy (Sulykos et al. 2017). This way the later effect may correspond to what is suggested by the predictive coding accounts (e.g., Stefanics et al. 2015). In the present study, however, the adjustment of the environmental model acquired by the standard and the incoming stimulus is highly improbable in either of the conditions.

Regarding the frontocentral region, we found that the pattern of the ERPs to the standard, deviant, and control stimuli as well as the difference waves is similar to the pattern at the posterior region but reversed and smaller. An additional analysis on peak and onset latencies with a fourlevel Anteriority factor (O1. Oz, O2 vs. PO3, POz, $\mathrm{PO} 4$ vs. $\mathrm{FC} 1, \mathrm{FCz}, \mathrm{FC} 2$ vs. F1, Fz, F2) found a main effect of Anteriority for peak latencies, $F(3,54)=4.85, p=0.005, \varepsilon=0.858$, $\eta_{\mathrm{p}}{ }^{2}=0.212$, but only the latency at the F sites was somewhat longer ( $p$ s between 0.003 and 0.053 compared to the other regions), and there was no difference for onset latencies, $F(3$, 54) $=0.29, p=0.834, \varepsilon=0.525$. Thus, the present data are not sufficient for distinguishing between a possible frontal source, a phase reversal, or use of the average reference.

As an unexpected finding, the SSA to the more complex snowflake pattern was larger than to the oblique bar pattern, which difference was observable for both peak amplitude and integrated activity at the posterior sites and for the integrated activity at the frontocentral sites. In addition, both the peak latency and the onset latency of the SSA were shorter in the case of the oblique bar pattern as standard at both regions. 
The scalp distributions for the control minus standard difference at the peak latencies differed, but the difference did not reach significance (Supplementary Information 4). For the oblique bar pattern as standard in the Deviant Snowflake Oddball condition, the ERP difference between the control and standard was confined to a narrow range. In this study, the stimuli did not elicit a detectable N1 component, but the lack of identified negativity in the N1 range does not exclude that superposition of various activities that cancelled each other in this range (Luck 2005). However, in the Deviant Bar Oddball condition (snowflake pattern as standard), the adaptation-related period included not only the N1, but also the $\mathrm{P} 2$ range. Therefore, it is difficult to argue that the standard-related effect is due to a simple refractoriness (or fatigue) of low-level input structures, reflected by the reduction of the N1 component. The long-lasting adaptation effect on the more complex stimulus argues against the possibility that the stronger adaptation was simply the consequence of an additive effect of low-level processes (e.g., the adaptation of various orientation-specific visual structures). It seems that in the case of complex stimuli SSA is a more powerful process than in the case of simple stimuli. Recently, Amado and Kovács (2016) reported data showing that adaptation/ repetition suppression fully explained the ERP difference between the deviant and standard for complex stimuli such as faces and chairs. We suggest that the adaptation difference in the present study was due to the faster build-up of the representation of a simple regular event (oblique bar pattern), as reflected by the latency of the control minus standard difference potential. The robust adaptation for complex stimuli (the snowflake pattern as standard), on the other hand, is an indicator of the build-up of a more elaborated representation of the standard.

One limitation of the present study is that the traditional oddball paradigm is not particularly optimal for investigating stimulus-specific adaptation (SSA). In this respect, the reversal of the deviant-standard relationship within short sequences (roving standard paradigm) is a more promising method (e.g., Baldeweg et al. 2004). Furthermore, a comparison of the deviant-related effects in the oddball paradigm and in paradigms developed for investigating adaptation is a more direct possibility (Bodnar et al. 2017). Another limitation in the present design is that it is impossible to compare the precise level of adaptation in the two equiprobable conditions for the oblique line and the snowflake stimuli. This difference may contribute to the difference between the deviant minus standard difference potentials and the deviant minus control difference potentials in the oblique line vs. snowflake conditions.

\section{Conclusions}

The visual mismatch negativity (vMMN), an event-related potential (ERP) difference between the deviant stimuli from oddball sequences and the corresponding identical stimuli from equiprobable sequences, was fairly similar for a simple deviant within a sequence of complex events and a complex deviant within a sequence of simple events. VMMN in the present design indicates that this activity does not require the adjustment of a predictive model and the incoming activity, and vMMN is not directly connected to orientation processes, i.e., it was not larger for the more salient stimulus. Stimulus-specific adaptation is stronger for complex stimuli. The adaptation difference is likely to indicate differences in the automatic acquisition of the representation of simple and more complex events.

Acknowledgements Open access funding provided by MTA Research Centre for Natural Sciences (MTA TTK). We thank Emese Várkonyi for the technical assistance.

Funding This work was supported by the Hungarian Scientific Research Fund [OTKA 119587].

\section{Compliance with ethical standards}

Conflict of interest The authors declare that they have no conflict of interest.

Ethical approval All procedures performed in studies involving human participants were in accordance with the ethical standards of the institutional and/or national research committee and with the 1964 Helsinki declaration and its later amendments or comparable ethical standards.

OpenAccess This article is distributed under the terms of the Creative Commons Attribution 4.0 International License (http://creativeco mmons.org/licenses/by/4.0/), which permits unrestricted use, distribution, and reproduction in any medium, provided you give appropriate credit to the original author(s) and the source, provide a link to the Creative Commons license, and indicate if changes were made.

\section{References}

Amado C, Kovács G (2016) Does surprise enhancement or repetition suppression explain visual mismatch negativity? Eur J Neurosci 43:1590-1600. https://doi.org/10.1111/ejn.13263

Astikainen P, Lillstrang E, Ruusuvirta T (2008) Visual mismatch negativity for changes in orientation-a sensory memorydependent response: visual mismatch negativity reflects sensory memory. Eur J Neurosci 28:2319-2324. https://doi.org/10.111 $1 / \mathrm{j} .1460-9568.2008 .06510 . \mathrm{x}$

Auksztulewicz R, Friston K (2016) Repetition suppression and its contextual determinants in predictive coding. Cortex 80:125-140. https://doi.org/10.1016/j.cortex.2015.11.024

Baldeweg T, Klugman A, Gruzelier J, Hirsch SR (2004) Mismatch negativity potentials and cognitive impairment in schizophrenia. 
Schizophr Res 69:203-217. https://doi.org/10.1016/j.schre s.2003.09.009

Bendixen A (2014) Predictability effects in auditory scene analysis: a review. Front Neurosci. https://doi.org/10.3389/fnins.2014.00060

Bodnar F, File D, Sulykos I, Kecskes-Kovács K, Czigler I (2017) Automatic change detection in vision: adaptation, memory mismatch, or both? II: oddball and adaptation effects on event-related potentials. Atten Percept Psychophys 8:2396-2411. https://doi. org/10.3758/s13414-017-1402-x

Clifford A, Holmes A, Davies IRL, Franklin A (2010) Color categories affect pre-attentive color perception. Biol Psychol 85:275-282. https://doi.org/10.1016/j.biopsycho.2010.07.014

Czigler I, Weisz J, Winkler I (2006) ERPs and deviance detection: visual mismatch negativity to repeated visual stimuli. Neurosci Lett 401:178-182. https://doi.org/10.1016/j.neulet.2006.03.018

Delorme A, Makeig S (2004) EEGLAB: an open source toolbox for analysis of single-trial EEG dynamics including independent component analysis. J Neurosci Methods 134:9-21. https://doi. org/10.1016/j.jneumeth.2003.10.009

Delorme A, Rousselet GA, Macé MJ-M, Fabre-Thorpe M (2004) Interaction of top-down and bottom-up processing in the fast visual analysis of natural scenes. Cogn Brain Res 19:103-113. https:// doi.org/10.1016/j.cogbrainres.2003.11.010

File D, File B, Bodnár F, Sulykos I, Kecskés-Kovács K, Czigler I (2017) Visual mismatch negativity (vMMN) for low- and highlevel deviances: a control study. Atten Percept Psychophys 79:2153-2170. https://doi.org/10.3758/s13414-017-1373-y

Friston K (2010) The free-energy principle: a unified brain theory? Nat Rev Neurosci 11:127-138. https://doi.org/10.1038/nrn2787

Garrido MI, Kilner JM, Stephan KE, Friston KJ (2009) The mismatch negativity: a review of underlying mechanisms. Clin Neurophysiol 120:453-463. https://doi.org/10.1016/j.clinph.2008.11.029

Gibson JJ (1937) Adaptation with negative after-effect. Psychol Rev 44:222-244. https://doi.org/10.1037/h0061358

Gonsalves BD, Kahn I, Curran T, Norman KA, Wagner AD (2005) Memory strength and repetition suppression: multimodal imaging of medial temporal cortical contributions to recognition. Neuron 47:751-761. https://doi.org/10.1016/j.neuron.2005.07.013

Grill-Spector K, Henson R, Martin A (2006) Repetition and the brain: neural models of stimulus-specific effects. Trends Cogn Sci 10:14-23. https://doi.org/10.1016/j.tics.2005.11.006

Guthrie D, Buchwald JS (1991) Significance testing of difference potentials. Psychophysiology 28:240-244. https://doi. org/10.1111/j.1469-8986.1991.tb00417.x

Jacobsen T, Schröger E (2001) Is there pre-attentive memory-based comparison of pitch? Psychophysiology 38:723-727. https://doi. org/10.1111/1469-8986.3840723

Karniski W, Blair RC, Snider AD (1994) An exact statistical method for comparing topographic maps, with any number of subjects and electrodes. Brain Topogr 6:203-210. https://doi.org/10.1007/ BF01187710

Kimura M (2012) Visual mismatch negativity and unintentional temporal-context-based prediction in vision. Int J Psychophysiol 83:144-155. https://doi.org/10.1016/j.ijpsycho.2011.11.010

Kimura M, Takeda Y (2013) Task difficulty affects the predictive process indexed by visual mismatch negativity. Front Hum Neurosci. https://doi.org/10.3389/fnhum.2013.00267

Kimura M, Takeda Y (2015) Automatic prediction regarding the next state of a visual object: electrophysiological indicators of prediction match and mismatch. Brain Res 1626:31-44. https://doi. org/10.1016/j.brainres.2015.01.013

Kimura M, Katayama J, Ohira H, Schröger E (2009) Visual mismatch negativity: new evidence from the equiprobable paradigm. Psychophysiology 46:402-409. https://doi.org/10.111 1/j.1469-8986.2008.00767.x
Kimura M, Ohira H, Schröger E (2010) Localizing sensory and cognitive systems for pre-attentive visual deviance detection: an sLORETA analysis of the data of Kimura et al. (2009). Neurosci Lett 485:198-203. https://doi.org/10.1016/j.neulet.2010.09.011

Kimura M, Schröger E, Czigler I (2011) Visual mismatch negativity and its importance in visual cognitive sciences. NeuroReport 22:669-673. https://doi.org/10.1097/WNR.0b013e32834973ba

Kimura M, Kondo H, Ohira H, Schroger E (2012) Unintentional temporal context-based prediction of emotional faces: an electrophysiological study. Cereb Cortex 22:1774-1785. https://doi. org/10.1093/cercor/bhr244

Kreegipuu K, Kuldkepp N, Sibolt O, Toom M, Allik J, Näätänen R (2013) vMMN for schematic faces: automatic detection of change in emotional expression. Front Hum Neurosci. https:// doi.org/10.3389/fnhum.2013.00714

Krekelberg B, Boynton GM, van Wezel RJA (2006) Adaptation: from single cells to BOLD signals. Trends Neurosci 29:250-256. https ://doi.org/10.1016/j.tins.2006.02.008

Kremláček J, Kreegipuu K, Tales A, Astikainen P, Põldver N, Näätänen R, Stefanics G (2016) Visual mismatch negativity (vMMN): a review and meta-analysis of studies in psychiatric and neurological disorders. Cortex 80:76-112. https://doi.org/10.1016/j.corte x.2016.03.017

Lafontaine MP, Lacourse K, Lina J-M, McIntosh AR, Gosselin F, Théoret H, Lippé S (2016) Brain signal complexity rises with repetition suppression in visual learning. Neuroscience 326:1-9. https://doi.org/10.1016/j.neuroscience.2016.03.059

Luck SJ (2005) An introduction to the event-related potential technique. MIT Press, Cambridge

Miller J, Patterson T, Ulrich R (1998) Jackknife-based method for measuring LRP onset latency differences. Psychophysiology 35:99-115. https://doi.org/10.1111/1469-8986.3510099

Näätänen R (1992) Attention and brain function. Erlbaum, Hillsdale

Näätänen R, Kujala T, Winkler I (2011) Auditory processing that leads to conscious perception: a unique window to central auditory processing opened by the mismatch negativity and related responses: auditory processing that leads to conscious perception. Psychophysiology 48:4-22. https://doi.org/10.111 1/j.1469-8986.2010.01114.x

O'Shea RP (2015) Refractoriness about adaptation. Front Hum Neurosci. https://doi.org/10.3389/fnhum.2015.00038

Paavilainen P, Arajärvi P, Takegata R (2007) Preattentive detection of nonsalient contingencies between auditory features. NeuroReport 18:159-163. https://doi.org/10.1097/WNR.0b013e328010e2ac

Sawamura H, Orban GA, Vogels R (2006) Selectivity of neuronal adaptation does not match response selectivity: a single-cell study of the fMRI adaptation paradigm. Neuron 49:307-318. https://doi. org/10.1016/j.neuron.2005.11.028

Sayres R, Grill-Spector K (2006) Object-selective cortex exhibits performance-independent repetition suppression. J Neurophysiol 95:995-1007. https://doi.org/10.1152/jn.00500.2005

Schröger E, Wolff C (1996) Mismatch response of the human brain to changes in sound location. Neuroreport 7:3005-3008

Sokolov EN (1963) Higher nervous functions: the orienting reflex. Annu Rev Physiol 25:545-580. https://doi.org/10.1146/annur ev.ph.25.030163.002553

Statsoft, Inc. (2016) STATISTICA, v. 13. Available from: http://www. statsoft.com/Products/STATISTICA-Features

Stefanics G, Czigler I (2012) Automatic prediction error responses to hands with unexpected laterality: an electrophysiological study. NeuroImage 63:253-261. https://doi.org/10.1016/j.neuroimage .2012 .06 .068

Stefanics G, Kimura M, Czigler I (2011) Visual mismatch negativity reveals automatic detection of sequential regularity violation. Front Hum Neurosci. https://doi.org/10.3389/fnhum.2011.00046 
Stefanics G, Kremláček J, Czigler I (2014) Visual mismatch negativity: a predictive coding view. Front Hum Neurosci 8:666. https://doi. org/10.3389/fnhum.2014.00666

Stefanics G, Astikainen P, Czigler I (2015) Visual mismatch negativity (vMMN): a prediction error signal in the visual modality. Front Hum Neurosci. https://doi.org/10.3389/fnhum.2014.01074

Sulykos I, Gaál ZA, Czigler I (2017) Visual mismatch negativity to vanishing parts of objects in younger and older adults. PLoS One 12:e0188929. https://doi.org/10.1371/journal.pone.0188929

Sulykos I, Gaál ZA, Czigler I (2018) Automatic change detection in older and younger women: a visual mismatch negativity study. Gerontology 64:318-325. https://doi.org/10.1159/000488588

Ulrich R, Miller J (2001) Using the jackknife-based scoring method for measuring LRP onset effects in factorial designs. Psychophysiology 38:816-827. https://doi.org/10.1017/S0048577201000610

Vogel BO, Shen C, Neuhaus AH (2015) Emotional context facilitates cortical prediction error responses: emotion-predictive coding interaction. Hum Brain Mapp 36:3641-3652. https://doi. org/10.1002/hbm. 22868
Winkler I (2007) Interpreting the mismatch negativity. J Psychophysiol 21:147-163. https://doi.org/10.1027/0269-8803.21.34.147

Winkler I, Czigler I (1998) Mismatch negativity: deviance detection or the maintenance of the "standard". NeuroReport 9:3809-3813. https://doi.org/10.1097/00001756-199812010-00008

Winkler I, Schröger E (2015) Auditory perceptual objects as generative models: setting the stage for communication by sound. Brain Lang 148:1-22. https://doi.org/10.1016/j.bandl.2015.05.003

Yu M, Li Y, Mo C, Mo L (2017) Newly learned categories induce pre-attentive categorical perception of faces. Sci Rep. https://doi. org/10.1038/s41598-017-14104-6

Publisher's Note Springer Nature remains neutral with regard to jurisdictional claims in published maps and institutional affiliations. 